\title{
Comparison of Stone Retrieval Basket, Stone Cone and Holmium Laser: Which One Is Better in Retropulsion and Stone-Free Status for Patients with Upper Ureteral Calculi?
}

\author{
Farzad Allameh ${ }^{1}{ }^{\circledR}$, Mohammadreza Razzaghi $^{2}$, Morteza Fallah-Karkan $^{2}{ }^{\circledR}$, Behnam Hosseini $^{\circledR}{ }^{\circledR}$, Ali Tayyebi \\ Azar $^{4}{ }^{\circledR}$, Arash Ranjbar ${ }^{2}{ }^{\oplus}$, Amir Hossein Rahavian ${ }^{2}$, Saleh Ghiasy ${ }^{2,5 *}$ \\ ${ }^{1}$ Center of Excellence for Training Laser Applications in Medicine, Shohada-e-Tajrish Hospital, Shahid Beheshti University of \\ Medical Sciences, Ministry of Health, Tehran, Iran \\ ${ }^{2}$ Laser Application in Medical Sciences Research Center, Shohada-e-Tajrish Hospital, Shahid Beheshti University of Medical \\ Sciences, Tehran, Iran \\ ${ }^{3}$ Akhtar Hospital, Shahid Beheshti University of Medical Sciences, Tehran, Iran \\ ${ }^{4}$ Nephrology and Kidney Transplant Research Center, Urmia University of Medical Sciences, Urmia, Iran \\ ${ }^{5}$ Department of Urology, Shohada-e-Tajrish Hospital, Shahid Beheshti University of Medical Sciences, Tehran, Iran
}

\section{*Correspondence to \\ Saleh Ghiasy, Urology Resident, Department of Urology, Shohada- e-Tajrish hospital, ShahidBeheshti University of Medical Sciences, Tehran, Iran Tel: +989128198037 ; Fax: +98 2122736386 Email: saleh.ghiasy@sbmu.ac.ir}

Published online July 6, 2019

\begin{abstract}
Introduction: Transurethral lithotripsy (TUL) is an appropriate treatment for ureteral stones and is usually used for stones in the middle and lower part of the ureter. Different devices such as the Holmium laser, the stone basket, and the stone cone exist to prevent any fragments from retropulsion during TUL. The present study aims to compare the advantages and disadvantages of the Holmium laser, the stone basket, and the stone cone.

Methods: A retrospective study was conducted from September 2016 to January 2018 comparing various TUL methods in 88 subjects with proximal ureteral calculi. The study participants were divided into 4 matched groups. The first one included 20 patients undergoing TUL with no device (group 1), the second group included 22 patients undergoing TUL while using the stone retrieval basket, the third group included 18 patients undergoing TUL while utilizing the stone cone and the fourth group included 28 patients undergoing TUL while using the Hol-YAG laser. Results: A residual stone $\geq 3 \mathrm{~mm}$ was recorded in $15.9 \%$ of the patients. The stone free rate was seen in $100 \%, 90.9,83.3 \%$, and $55 \%$ of the Holmium laser group, the retrieval basket group, the stone cone group and the no device group respectively $(P=0.001)$. The lowest rate of surgery complications including ureteral perforation, post-operative fever, and mucosal damage between the 4 groups $(P=0.003)$ and the highest time of surgery $(P=0.001)$ belonged to the laser group. If we want to ignore the laser group, the success rate for lithotripsy was better in both groups with a stone retrieval device compared to the no device group, but no advantage existed between the stone basket and the stone cone.

Conclusion: We can safely conclude that lasers significantly help to prevent stone migration during TUL. If we want to ignore the laser group, the success rate for lithotripsy was significantly better in both groups with a stone retrieval device compared to the no device group, but no advantage existed between the stone basket and the stone cone.

Keywords: Stone cone; Stone retrieval basket; Transurethral lithotripsy; Ureteral calculi; Ureteral stone; Lithoclast lithotripter, Holmium-YAG laser.
\end{abstract}

\section{Introduction}

Ureteral calculi, as a routine urological practice and a common condition, affects people at all ages and helps in treating cases with urolithiasis. ${ }^{1,2}$ Ureteral stones have many complications such as obstructive uropathy and subsequent deterioration of renal function. ${ }^{3}$ Several factors are involved in choosing a suitable management option for ureteral stones, including the stone location and its size and composition. Other factors may include equipment availability, patient characteristics, and even surgeons' capabilities. ${ }^{4}$ Transurethral lithotripsy (TUL) is a frequent treatment for ureteral stones, and is usually used for middle and lower uretercalculi. ${ }^{5}$ The major advantage of ureteroscopy is direct visualization of the 
ureter, enabling observation and management of stones. ${ }^{6}$ Various sources of energy are used for endoscopic lithotripsy, including laser, pneumatic, ultrasonic, and electrohydraulic lithotripters; however, pneumatic lithotripters is the most common source. ${ }^{7,8}$ It has been commonly used to treat ureteral stones mostly owing to its low price and high effectiveness. One of the disadvantages of this method is the migration of fragments during TUL, especially in upper ureteral stones.9-11 In this regard, different devices such as the stone retrieval basket and the stone cone are used to prevent fragments retropulsion during TUL.,12 The basket catheter device was used to prevent the stone from migrating to the renal pelvis during the TUL procedure. ${ }^{13}$ The stone cone, as a new device, consists of an inner wire and an outer catheter; the inner wire is formed by the distal floppy tip, the cone, and the proximal wire. The cone can help the surgeon to hold the top stone during lithotripsy. ${ }^{14}$

Nowadays, the holmium: yttrium-aluminum-garnet (Ho:YAG) laser is used for different urologic surgeries such as treatment of urinary calculi. Compared with other pieces of equipment, the laser has a lower complication rate and a high stone free rate (SFR) but because of high cost and low access, it is not applied to each patient in every institute. ${ }^{15}$

In this retrospective investigation, we compared the outcome of the stone basket, the stone cone and the Ho:YAG laser in TUL of ureteral calculi.

\section{Methods \\ Sampling \\ Eight hundred ninety-six patients diagnosed with ureteral calculi from September 2016 to January 2018 were hospitalized in Shohada-e-Tajrish Hospital, Shahid Beheshti University of Medical Sciences, Tehran, Iran. After exclusion of lower and middle ureteral stones $(\mathrm{n}=359), 159$ patients with pneumatic lithotripter (PL) and laser lithotripter were enrolled. The exclusion criteria were kidney anomalies, severe musculoskeletal deformity, history of ureteral obstruction, positive urinary culture, and pregnancy. We included patients aged $>18$ with upper ureteral stones.}

A total of 22 TUL procedures were conducted using the stone retrieval basket. Eighteen patients underwent TUL while utilizing the stone cone and 28 patients underwent TUL while using the Hol-YAG laser. Therefore, from the rest of patients who had ureteral calculi and underwent TUL with pnematic lithotripter without using any extra device, we found 20 matched patients.

\section{Study Protocol}

For all cases, a checklist including demographic characteristics, clinical and stone features, intra- and post-operative data was filled.

A pre-operative urinary analysis and culture, a renal function test and a non-contrast abdominopelvic computed tomography scan (NCCTS) were conducted for all the patients before intervention.

Semi-rigid ureteroscope 9.5 Fr (Wolf Inc., Germany) was used in all the procedures, and lithotripsies were performed under direct endoscopic vision without the pre-stented procedure. Ureter was accessed using 0.035 inch guide wires. The $0.8 \mathrm{~mm}$ to $1.2 \mathrm{~mm}$ lithotripter (Swiss lithoclast) was passed through the ureteroscope working channel. The tip of probe was rested on the calculi surface, and the probe was activated under 2.5 atm in either a continuous pulse or a single shut mode.

Stone Cone ${ }^{\mathrm{TM}}$ Nitinol Retrieval Coil and OptiFlex ${ }^{\mathrm{TM}}$ 1.3Fr Nitinol Stone Retrieval Basket (Boston Scientific Corp., Natick, MA, USA) were used in our center. We used a Ho:YAG laser (manufactured in Iran). After the stone was visualized, the laser fiber $(500 \mathrm{Mm})$ was crossed through the working channel of the ureteroscope and then the laser was discharged. Laser Lithotripsy was done until the stones were fragmented and became as small as sand particles. Ureteroscopy combined with the Ho: YAG laser was done by a single urologist.

Double J (DJ) stent was inserted in cases with difficult dilation, a prolonged procedure and a residual stone. Ten days after the procedure, the stent was removed from the patients whose stone free status was achieved. If there were any perforation in ureter, DJ was removed after 6 weeks.

All the procedures were conducted under spinal anesthesia and lithotomy position. Single dose prophylactic intravenous antibiotics (routinely single dose intravenous ciprofloxacin $400 \mathrm{mg}$ ) were administered before surgery.

\section{Primary and Secondary End Points}

The primary outcomes considered the SFR and the rate of pushing back between the groups; however, the long-term complications due to the retrospective nature of the study could not be evaluated.

SFR definitions in this study were the absence of particles over $3 \mathrm{~mm}$ in post-operative kidney, ureter, and bladder radiography in radio-opaque calculi and NCCTS just in the radiolucent stone because of cost saving.

The secondary end points were preoperative and postoperative complications, including the ClavienDindo modified classification. ${ }^{15}$

\section{Statistical Analysis}

Statistical package for social sciences (v.21, IBM Inc., Chicago, IL, USA) software was used to analyze the data. While quantitative variables were described as mean and standard deviation, qualitative variables were described as frequency and percent. All the quantitative variables were compared in the subgroups by independent samples $t$ test, and all the nominal variables were compared by 
chi-square test. $P$ values $\leq .05$ were considered statistically significant.

\section{Results}

The demographic characteristics and clinical data of the four groups were similar in terms of age, gender, laterality and mean size of stones (Table 1). Figure 1 shows 3 different pieces of equipment are needed. Table 2 presents the details of intra- and post-operation data. The lowest rate of surgery complications including ureteral perforation, Post-operative fever and mucosal damage between 4 groups $(P=0.003)$ and the highest time of surgery $(P=0.001)$ belonged to the laser group.

The SFRs seen in the laser group, the basket group, the stone cone group and the no device group were $100 \%, 90.9$, $83.3 \%$ and $55 \%$ respectively $(P=0.001)$. Stone migration was significantly higher in the no device group although there was no difference between the stone basket group and the stone cone one.

According to the Clavien-Dindo modified classification, 19 Grade II, 3 Grade IIIa and 1 Grade IIIb complications were seen. Three intraoperative ureteral perforations were successfully treated with a DJ stent placing, removed after 6 weeks. One subject in the stone cone group needed open surgery and ureteroureterostomy due to device malfunction. In this case, after lithotripsy, and during pulling out the device, the cone did not straighten and we had a $4-\mathrm{cm}$ ureteral perforation in the medial wall. Fortunately, avulsion did not occur (Table 3).

No long-term complication documented in the included cases was found. If we want to ignore the laser group, the success rate for lithotripsy was significantly higher in both groups with the stone retrieval device compared to the no device group, but no advantage existed between the stone basket and the stone cone.

In the no device group, nine patients did not gain an immediate stone-free status. Most of these patients in whom fragments were pushed back had a ureteral stone $\geq 1 \mathrm{~cm}$ (5 from 9), and one case with a stone embedded in ureteral edema. Three patients were managed by flexible ureteroscopy and retrograde intrarenal surgery in the same session, and 4 cases were managed by extracorporeal shock wave lithotripsy (ESWL) before removal of DJ stent respectively.

\section{Discussion}

TUL is proper management of ureteral calculi, which has increased over the last century; however, one of its vital disadvantages is stone migration during this method., ${ }^{9,17}$ Depending on previous studies, $40 \%$ of stone migration was reported from the proximal ureter. ${ }^{18}$ Over time, the development of TUL has stimulated growth in many different devices used to prevent stone migration.

This study analyzed the three different devices used during TUL in 88 subjects with ureteral calculi in the 4 groups. In our study, $100 \%$ of cases had an upper ureter stone, and we used TUL for their treatment; however, in many other studies, researchers used ESWL., In the current study, a $15.9 \%$ stone retropulsion was observed. Various methods such as the laser, the stone retrieval basket and the stone cone devices exist to prevent the migration of the fragments.

Farahat et $\mathrm{al}^{12}$ compared the stone cone and entrapment net to prevent the stone retropulsion during PL in 180 patients. They concluded that the use of the stone cone or N-Trap was valuable during ureteroscopic PL for the treatment of proximal ureteral stones. Both devices significantly decreased residual fragments $(P<0.05)$, the incidence of ureteral trauma, and the need for the auxiliary procedure. However, the stone cone was more effective in preventing proximal stone migration and subsequent SFR.

The stone retrieval basket is useful to extract the whole stone; however, in previous studies, it is usually used for stones smaller than $6 \mathrm{~mm} .{ }^{9}$ Furthermore, according to other studies, we know that a disadvantage of this device

Table 1. Baseline Characteristics in the 4 Groups

\begin{tabular}{|c|c|c|c|c|}
\hline Variable & Laser $(n=28)$ & Basket $(n=22)$ & Stone cone $(n=18)$ & No device $(n=20)$ \\
\hline Mean age $\pm S D, y$ & $48.4 \pm 10.7$ & $42.7 \pm 11.53$ & $49 \pm 10$ & $45.4 \pm 8.7$ \\
\hline Male, $\mathrm{n}$ & 17 & 13 & 11 & 11 \\
\hline Previous history of TUL, $\mathrm{n}$ & 1 & 2 & 1 & 1 \\
\hline \multicolumn{5}{|l|}{ Stone laterality } \\
\hline Right side, $\mathrm{n}$ & 13 & 10 & 11 & 8 \\
\hline Left side, $\mathrm{n}$ & 15 & 12 & 7 & 12 \\
\hline Stone diameter, $\mathrm{mm}$ & $8.5 \pm 1.2(7-11)$ & $8.7 \pm 1.7(9-12)$ & $8.7 \pm 1.8(8-13)$ & $8.6 \pm 1.7(8-11)$ \\
\hline Number of stones, $\mathrm{n}$ & $1.2 \pm 0.2$ & $1.2 \pm 0.7$ & $1.2 \pm 0.4$ & $1.2 \pm 0.2$ \\
\hline \multicolumn{5}{|l|}{ Duration of stone symptoms } \\
\hline$<2$ months, $\mathrm{n}$ & 21 & 19 & 17 & 16 \\
\hline$>2$ month, $\mathrm{n}$ & 7 & 3 & 1 & 4 \\
\hline
\end{tabular}




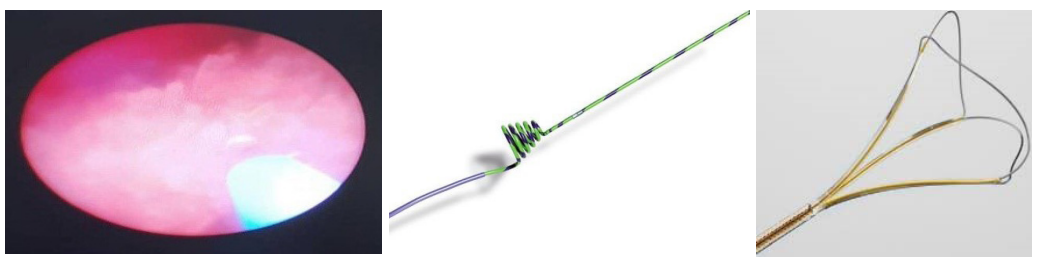

Figure 1. (A) Ho:YAG laser (manufactured in Iran). (B) Stone Cone ${ }^{\mathrm{TM}}$ Nitinol Retrieval Coil. (C) Nitinol Stone Retrieval Basket (Boston Scientific).

Table 2. Comparison of the Operation Variables Between the 4 Groups

\begin{tabular}{|c|c|c|c|c|c|}
\hline Variable & Laser $(n=28)$ & Basket $(n=22)$ & Stone Cone $(n=18)$ & No Device $(\mathrm{n}=\mathbf{2 0})$ & $\boldsymbol{P}$ \\
\hline \multicolumn{6}{|l|}{ Complications } \\
\hline Ureteral perforation, $\mathrm{n}$ & 0 & 1 & 2 & 1 & \multirow{3}{*}{0.003} \\
\hline Post-operative fever, $\mathrm{n}$ & 1 & 2 & 3 & 1 & \\
\hline Mucosal damage, $\mathrm{n}$ & 1 & 6 & 5 & 3 & \\
\hline Mean operation time $\pm \mathrm{SD}$, min & $48.7 \pm 10.7$ & $41.3 \pm 6.5$ & $44 \pm 7.6$ & $38.8 \pm 5.2$ & 0.001 \\
\hline Mean hospital stay $\pm \mathrm{SD}, \mathrm{h}$ & $27.2 \pm 7$ & $26.5 \pm 7.5$ & $26.8 \pm 5.6$ & $25.3 \pm 5.5$ & 0.7 \\
\hline Immediate stone-free status, No. (\%) & 28 & 20 & 15 & 11 & 0.001 \\
\hline Stone migration, No. (\%) & 1 & 1 & 1 & 7 & 0.002 \\
\hline
\end{tabular}

Table 3. Distributed Complication by Method

\begin{tabular}{|c|c|c|c|}
\hline \multirow{2}{*}{ Complication (Number of Patients) } & \multicolumn{3}{|c|}{ Method } \\
\hline & Basket & Stone Cone & No Device \\
\hline Number of patients with intraoperative ureteral perforations were successfully treated with a DJ stent placing & 1 & 1 & 1 \\
\hline Number of patients needed open surgery and ureteroureterostomy due to device malfunction. & - & 1 & - \\
\hline
\end{tabular}

is possible injury of the ureteral wall. ${ }^{14,19,20}$ The stone cone device is a newer piece of technology used instead of the stone retrieval basket; it helps to prevent the stone retropulsion. In addition, with the pneumatic energy, it can directly affect the stone center, causing it to fragment into pieces smaller than two mm. According to previous studies, we know that the pneumatic lithotripter cannot affect the calculi center in subjects not using the stone cone device, resulting in increased stone migration in these patients. ${ }^{9}$

The results indicated that the duration of the surgery in the group using the stone retrieval basket was lower than that in the laser and stone cone arms. According to previous studies and the present study, the duration of the surgery for the stone cone device was more than the surgery with the stone retrieval basket. ${ }^{1}$

Generally, the residual stone fragments $\geq 3 \mathrm{~mm}$ were $15.9 \%(n=14)$, with a $9.1 \%$ occurrence in the stone retrieval basket group, which was lower than the stone cone group (16.6\%). However, the residual fragments in both retrieval device groups compared to the no device group were significantly better.

Retrograde ureteroscopy has become a common procedure to manage urinary stones; however, the literature about major complications is still limited. ${ }^{21}$ According to other studies, we know that the stone cone device has a lower major mucosal abrasion than the stone retrieval basket device, but the flat wire basket by which patients after surgery recover quicker is more popular. ${ }^{1}$ In 2002, in one study comparing the stone retrieval basket device to the stone cone, the stone cone device was significantly more successful than the stone retrieval basket device ${ }^{14}$; however, it was more costly.

In this era, ureter stone treatment shifted from open surgery to less invasive interventions. Today, the laser has an excellent application in urologic diseases. ${ }^{22-24}$

Endoscopic laser lithotripsy (LL) is frequently used to manage patients with urolithiasis. ${ }^{8,25}$ A number of lasers have been used for LL, of which the Ho:YAG has become one of the most frequently used one. ${ }^{26}$ The advantages of the Ho:YAG lithotripter are as follows: it can pass through a rigid or flexible ureteroscope ${ }^{27}$; all types of stones with varying compositions can be fragmented ${ }^{28}$; and the probability of stone migration is lower. ${ }^{6}$ However, cost and inaccessibility of the laser led to limitations on the application of this device for all of patients, and we need to evaluate and recommend other devices such as the retrieval basket and the stone cone in this situation. ${ }^{15}$ 


\section{Limitations and Recommendations}

Limitations of this study include its retrospective nature and this inevitably introduces selection bias. The second limitation was a short-term follow-up and finally the small sample size.

\section{Conclusion}

We can safely conclude that lasers significantly helped to prevent stone migration during TUL. In addition to the laser in some situations, we can conclude that both of the stone retrieval basket and stone cone devices significantly helped to prevent the stone from pushing back during TUL. Nevertheless, the stone cone device had fewer complications than the stone retrieval basket device; however, the duration of the surgery and the amount of residual stones were lower in the groups using the stone retrieval basket in comparison with the stone cone device.

\section{Ethical Considerations}

As a retrospective study, no intervention has been made.

\section{Conflict of Interests}

The authors declare no conflict of interest.

\section{References}

1. Pardalidis NP, Papatsoris AG, Kosmaoglou EV. Prevention of retrograde calculus migration with the Stone Cone. Urol Res. 2005;33(1):61-64. doi:10.1007/s00240-004-0453-3

2. Abedi A, Razzaghi MR, Allameh F, Aliakbari M, Ranjbar A, Fallah Karkan M. Pneumatic Lithotripsy Versus Laser Lithotripsy for Ureteral Stones. J Lasers Med Sci. 2018;9(4):233-236. doi:10.15171/jlms.2018.42

3. Fallah Karkan M, Ghiasy S, Ranjbar A, Javanmard B. Evaluation of $200 \mathrm{Mm}, 365 \mathrm{Mm}$ and $500 \mathrm{Mm}$ Fibers of Ho: YAG Laser in Transurethral Lithotripsy of Ureteral: A Randomize Control Trial. J Lasers Med Sci. 2018;9(1):6972. doi:10.15171/jlms.2018.14

4. Elashry OM, Tawfik AM. Preventing stone retropulsion during intracorporeal lithotripsy. Nat Rev Urol. 2012;9(12):691-698. doi:10.1038/nrurol.2012.204

5. Shigemura $K$, Yasufuku $T$, Yamashita $M$, Arakawa $S$, Fujisawa M. Efficacy of combining flexible and rigid ureteroscopy for transurethral lithotripsy. Kobe J Med Sci. 2010;56(1):E24-28.

6. Abedi AR, Allameh F, Razzaghi MR, et al. The Efficacy and Safety of Laser Lithotripsy in Pregnancy. J Lasers Med Sci. 2017;8(2):84-87. doi:10.15171/jlms.2017.15

7. Rabani SM, Moosavizadeh A. Management of large proximal ureteral stones: a comparative clinical trial between transureteral lithotripsy (TUL) and shock wave lithotripsy (SWL). Nephrourol Mon. 2012;4(3):556-559. doi:10.5812/numonthly.3936

8. Razzaghi MR, Fallah Karkan M, Ghiasy S, Javanmard B. Laser application in iran urology: a narrative review. $J$ Lasers Med Sci. 2018;9(1):1-6. doi:10.15171/jlms.2018.01

9. Gonen $\mathrm{M}$, Cenker A, Istanbulluoglu O, Ozkardes H. Efficacy of dretler stone cone in the treatment of ureteral stones with pneumatic lithotripsy. Urol Int. 2006;76(2):159-
162. doi:10.1159/000090881

10. Sun Y, Wang L, Liao G, et al. Pneumatic lithotripsy versus laser lithotripsy in the endoscopic treatment of ureteral calculi. J Endourol. 2001;15(6):587-590. doi:10.1089/089277901750426346

11. Marguet CG, Sung JC, Springhart WP, et al. In vitro comparison of stone retropulsion and fragmentation of the frequency doubled, double pulse nd:yag laser and the holmium:yag laser. J Urol. 2005;173(5):1797-1800. doi:10.1097/01.ju.0000154341.08206.69

12. Farahat YA, Elbahnasy AE, Elashry OM. A randomized prospective controlled study for assessment of different ureteral occlusion devices in prevention of stone migration during pneumatic lithotripsy. Urology. 2011;77(1):30-35. doi:10.1016/j.urology.2010.05.063

13. Kressel K, Hoffmann H, Butz M. Long-term experience with transurethral rigid ureteroscopy as a complementary method to extracorporeal shockwave lithotripsy. Urol Int. 1992;48(1):76-80. doi:10.1159/000282301

14. Desai MR, Patel SB, Desai MM, et al. The Dretler stone cone: a device to prevent ureteral stone migration-the initial clinical experience. J Urol. 2002;167(5):1985-1988.

15. Gupta PK. Is the holmium:YAG laser the best intracorporeal lithotripter for the ureter? A 3-year retrospective study. J Endourol. 2007;21(3):305-309. doi:10.1089/end.2006.0247

16. Clavien PA, Barkun J, de Oliveira ML, et al. The ClavienDindo classification of surgical complications: five-year experience. Ann Surg. 2009;250(2):187-196. doi:10.1097/ SLA.0b013e3181b13ca2

17. Lopez M, Hoppe B. History, epidemiology and regional diversities of urolithiasis. Pediatr Nephrol. 2010;25(1):49 59. doi:10.1007/s00467-008-0960-5

18. Knispel HH, Klan R, Heicappell R, Miller K. Pneumatic lithotripsy applied through deflected working channel of miniureteroscope: results in 143 patients. J Endourol. 1998;12(6):513-515. doi:10.1089/end.1998.12.513

19. Dretler SP. The stone cone: a new generation of basketry. $J$ Urol. 2001;165(5):1593-1596.

20. Maislos SD, Volpe M, Albert PS, Raboy A. Efficacy of the Stone Cone for treatment of proximal ureteral stones. J Endourol. 2004;18(9):862-864. doi:10.1089/ end.2004.18.862

21. Cindolo L, Castellan P, Primiceri G, et al. Life-threatening complications after ureteroscopy for urinary stones: survey and systematic literature review. Minerva Urol Nefrol. 2017;69(5):421-431. doi:10.23736/s0393-2249.17.02787-4

22. Fallah Karkan M, Razzaghi MR, Javanmard B, Tayyebiazar A, Ghiasy S, Montazeri S. Holmium: YAG Laser Incision of Bladder Neck Contracture Following Radical Retropubic Prostatectomy. Nephrourol Mon. 2019;11(1):e88677. doi:10.5812/numonthly.88677

23. Fallah Karkan M, Razzaghi MR, Karami H, Ghiasy S, Tayyebiazar A, Javanmard B. Experience of 138 transurethral urethrotomy with holmium: YAG laser. J Lasers Med Sci. 2019;10(2):104-107. doi:10.15171/ jlms.2019.17

24. Ghiasy S, Fallah Karkan M, Razzaghi MR, Ranjbar A, Rahavian A, Javanmard B. Is Holmium Laser an Appropriate Modality to Treat Genital Warts? J Lasers Med Sci. 2018;10(1):70-74. doi:10.15171/jlms.2019.11 
25. Javanmard B, Fallah Karkan M, Razzaghi M, Ghiasy S, Ranjbar A, Rahavian A. Surgical management of vesical stones in children: a comparison between open cystolithotomy, percutaneous cystolithotomy and transurethral cystolithotripsy with holmium-YAG Laser. J Lasers Med Sci. 2018;9(3):183-187. doi:10.15171/ jlms.2018.33

26. Lee J, Gianduzzo TR. Advances in laser technology in urology. Urol Clin North Am. 2009;36(2):189-198. doi:10.1016/j.ucl.2009.02.004

27. Geavlete P, Multescu R, Georgescu D. [Flexible ureteroscopic approach in upper urinary tract pathology]. Chirurgia (Bucur). 2006;101(5):497-503.

28. Teichman JM, Vassar GJ, Bishoff JT, Bellman GC. Holmium: YAG lithotripsy yields smaller fragments than lithoclast, pulsed dye laser or electrohydraulic lithotripsy. $J$ Urol. 1998;159(1):17-23. 